\title{
Über Caseinokyrin.
}

\section{Mitteilung.}

Von

M. Siegfried.

(Aus der chemischen Abteilung des physiologischen Instituts Leipzig.)

(Uer Redaktion zugegangen am 6. November 1906.)

I. Bestätigung der Einheitlichkeit des Caseinokyrins durch Untersuchung des Phosphorwolframates.

Vor kurzem haben Zd. H. Skraup und Witt ${ }^{1}$ ) Casein in der von mir ${ }^{2}$ ) für die Darstellung des Caseinokyrins angegebenen Weise hydrolysiert, auch die Phosphorwolframsäurefällung des Rohproduktes nach der von mir gegebenen Vorschrift durch Zersetzung mit Baryt nach vorherigem Lösen in verdünntem Ammoniak zersetzt. In Übereinstimmung mit mir finden die Verfasser, daß das aus dieser Phosphorwolframsäurefällung dargestellte Rohprodukt nicht einheitlich ist. Einen Teil dieses Rohproduktes haben Skraup und Witt zweimal nach Lösen in verdünnter Schwefelsäure mit Alkohol gefällt und dies so erhaltene Sulfat weiter untersucht. Es ergab sich, daß dieses Sulfat kein reines Kyrinsulfat war, daß es Lysin enthielt, und daß die Phosphorwolframate nicht völlig in $80 \%$ igem Alkohol löslich waren. Ferner war ein Teil der aus den Phosphorwolframaten dargestellten Substanz nach schwachem Ansäuern mit Jodwasserstoffsäure durch Kaliumquecksilberjodid fällbar. Da ich in der eben zitierten Arbeit durch zweimaliges Fällen aus verdünnter Schwefelsäure in verdünnten Alkohol ein Kyrinsulfat erhalten habe, das bei weiterem Umfällen seine Zusammensetzung nicht mehr änderte, und da auch Skraup und Witt zweimal aus S. 463 .

1) Sitzungsbericht d. Kais. Akad. d. Wissensch., Wien 1906, Bd. CXV,

2) Diese Zeitschrift, Bd. XLIII, S. 46. 
verdünnter Schwefelsäure mit Alkohol gefällt haben, machen sie die Annahme, daß ihr Sulfat mit dem meinigen identisch gewesen sei. Auf Grund dieser Annahme schließen sie: weil ihr Sulfat nicht rein war, ist auch das von mir dargestellte Sulfat nicht einheitlich gewesen und deshalb ist die Existenz des Kyrins nicht bewiesen.

Aus dem Umstande, daß Skraup und Witt das Rohprodukt zweimal gefällt hatten, wären sie auch dann nicht zu der Annahme, ihr Sulfat sei mit dem meinigen identisch, berechtigt gewesen, wenn sie die Umfällung ebenso ausgeführt hätten wie ich; denn es wäre notwendig gewesen, wenigstens festzustellen, ob ihr Sulfat auch die von mir für das Caseinokyrinsulfat ermittelte Zusammensetzung besaß. Aber Skraup und Witt haben nicht einmal in derselben Weise wie ich umgefällt, sondern unter anderen Bedingungen und unter Verwendung von viel weniger Alkohol und Schwefelsäure. Ich habe angegeben: ${ }^{1}$ ) «Die Umfällung geschieht am besten aus 5\% iger Schwefelsäure in Alkohol, wobei etwa auf $15 \mathrm{ccm}$ der schwefelsauren Lösung 11 Alkohol absolut zu nehmen ist. Vorteilhaft fällt man das Rohsulfat zunächst einige Male als Öl durch allmählichen Zusatz von Alkohol zu der schwefelsauren Lösung des Kyrins. Selbstverständlich ist nur die Prüfung des Sulfates, namentlich die Elementaranalyse maßgebend dafür, ob das Sulfat rein oder noch weiter umzufällen ist.» Bei dem von mir zweimal gefällten Sulfat, auf welches sich Skraup und Witt beziehen, ist die zweite Fällung, wie an der betreffenden Stelle angegeben, ${ }^{2}$ ) in folgender Weise ausgeführt worden: «Die Lösung von $22 \mathrm{~g}$ «büchsentrockenem.


verrührt. Nach der Fällung wurde noch 1 Stunde weiter gerührt.’

Skraup und $\mathrm{Witt}^{3}$ ) hingegen sind folgenderweise verfahren : «Es wurden $70 \mathrm{~g}$ des Sirups in $96 \mathrm{ccm} 10 \%$ iger Schwefelsäure gelöst, je $35 \mathrm{ccm}$ in einer Flasche mit $600 \mathrm{ccm}$ absoluten Alkohols in Anteilen vermischt und auf der Maschine geschüttelt.

1) 1. c. S. 48 .

2) 1. c. S. 51 . S. 470 .

s) Sitzungsbericht d. Kaiserl. Akad. d. Wissensch., 1906, Bd. CXV, 
Die Flüssigkeit wurde bald klar. Das an der Wand harzig ausgeschiedene Sulfat, je $29 \mathrm{~g}$, wurde durch Schütteln in $25 \mathrm{ccm}$ $10 \%$ iger Schwefelsäure gelöst und wieder $600 \mathrm{ccm}$ alsoluten Alkohols zugefügt.»

Bei der von mir vorgeschriebenen Fällung durch Eingießen oder Eintropfenlassen der schwefelsauren Lösung in Alkohol unter Rühren wird das Sulfat in kleinen, mikroskopischen Körnchen ausgeschieden, die nach dem Auswaschen mit Alkohol und Äther und Trocknen über Schwefelsäure ein Pulver darstellen. Die von Skraup und Witt vorgenommene Fällung liefert eine harzige Ausscheidung. Diese Art Fällung ist zu vergleichen mit der vorhin erwähnten Ölfällung. Vergleichende Versuche, die im hiesigen Laboratorium mit Ölfällungen einerseits und der Fällung durch Eingießen der Sulfatlösung in viel Alkohol unter kräftigem Umrühren bei anderen Kyrinen angestellt worden sind, haben die große Überlegenheit der zweiten Art über die erstere ergeben (s. z. B. die vorhergehende Mitteilung von $\mathrm{H}$. Kirbach). Hiernach sind Ölfällungen nicht vorteilhaft.

Anmerkung: Der so sehr verschiedene Effekt der einen und anderen Art der Fällungsweisen bei den Kyrinen dürfte auch bei anderen Körpern zutage treten. Wir haben auch früher stets die Peptone durch Einrühren ihrer Lösung in viel Alkohol gereinigt. Eine genaue Angabe der Art des Umfällens dürfte daher in jedem Falle erwünscht sein, während man häufig nur die Angabe findet, die Substanzen seien so und so oft umgefällt worden.

Die Tatsache, daß Skraup und Witt zu der Annahme, das von ihnen untersuchte Sulfat sei identisch mit Kyrinsulfat, nicht berechtigt waren, macht die Schlüsse, welche die Verfasser aus den Ergebnissen der Untersuchung ihres Sulfates auf das Caseinokyrinsulfat gezogen haben, hinfällig.

Die erwähnte Mitteilung von Skraup und Witt ergab. die Möglichkeit, von neuem die Einheitlichkeit des Caseinokyrins zu prüfen. Die Verfasser haben auf einem neuen Wege gezeigt, wie sich erkennen läßt, daß das Gemisch von Produkten, die sich durch Einwirkung von Salzsäure auf Casein bei Körpertemperatur bilden und durch Phosphorwolframsäure fällbar sind, nicht einheitlich ist. Die Anwendung des von Skraup und Witt eingeschlagenen Weges auf Kyrinsulfat mußte somit erkennen 
lassen, ob dieses frei von den im Rohsulfat vorhandenen Verunreinigungen ist.

$7,5 \mathrm{~g}$ nach einer Trockenbestimmung bei $95^{\circ}$ als trocken berechnetes Kyrinsulfat wurden in $500 \mathrm{ccm}$ Wasser gelöst. Die Lösung war im Gegensatz zu der Lösung des Sulfates von Skraup und Witt ganz klar. Sie wurde durch $120 \mathrm{ccm}$ einer Lösung, welche $100 \mathrm{~g}$ Phosphorwolframsäure in $220 \mathrm{ccm}$ enthielt, ausgefällt. Nach Absaugen und Trocknen auf dem Wasserbade wog das Phosphorwolframat $52 \mathrm{~g}$. Dieses wurde mit $87 \mathrm{ccm} 80 \%$ igen Alkohols bei gewöhnlicher Temperatur verrührt; nach 4 Stunden hatte es sich völlig gelöst. Die Lösung wurde auf einer kleinen Filterscheibe durchgesaugt, dieselbe

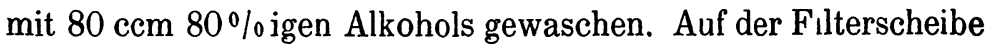
fand sich kein ungelöstes Phosphorwolframat. Aus $97 \mathrm{~g}$ Phosphorwolframat haben Skraup und Witt $3 \mathrm{~g}$ eines in $80 \%$ igem Alkohol unlöslichen Phosphorwolframates erhalten. Diese Verunreinigung fand sich im Kyrinphosphorwolframat also ebenfalls nicht vor.

Aus der alkoholischen Lösung wurde das Phosphorwolframat durch $340 \mathrm{ccm}$ Wasser ausgeschieden. Unter dem Mikroskope zeigte es sich aus gleichmäßigen, kleinen, durchsichtigen Körnchen bestehend; Nadeln oder Drusen waren nirgends zu sehen. Auch nach Lösen durch Erwärmen des mit Deckglas bedeckten Objektträgers schied sich das Phosphorwo!framat beim Erkalten lediglich in kleinen Körnchen aus. Nach Absaugen am folgenden Tage und Trocknen auf dem Wasserbade wog dieses Phosphorwolframat $44,5 \mathrm{~g}$. Nach Übergießen mit $100 \mathrm{ccm} 80 \%$ igen Alkohols halte es sich innerhalb 5 Stunden bis auf eine geringe Menge gröberer zusammengebackter Körnchen gelöst. Nach Absaugen auf kleiner Filterscheibe, Nachwaschen mit etwas 80\% igem Alkohol und Trocknen der Filterscheibe auf dem Wasserbade betrug das Gewicht des ungelösten Phosphorwolframates $0,105 \mathrm{~g}$. Wenn dies wirklich von einer Verunreinigung herrührte, die bei dem ersten Lösen des Phosphorwolframates mit in Lösung gegangen wäre, so betrüge ihre Menge nur ca. $0,24 \%$ vom ganzen Phosphorwo:framate.

Das a koholische Filtrat wurde mit der doppelten Menge 
Wassers vermischt. Es schied sich das Phosphorwolframat wieder in gleichmäßigen kleinen mikroskopischen Körnchen aus. Anderntags abgesaugt; Gewicht des auf dem Wasserbade getrockneten Phosphorwolframates $39 \mathrm{~g}$.

Aus dem Filtrate von der ersten Umfällung des Phosphorwolframates aus $80 \%$ igem Alkohol durch Wasser wurde durch Zusatz bei gewöhnlicher Temperatur gesättigten Barytwassers es genügten $150 \mathrm{ccm}$ - die Phosphorwolframsäure ausgefällt, das Filtrat nach Zusatz von Ammoncarbonat und Filtrieren eingedampft. Der Rückstand wog 0,402 g. Er wurde in möglichst wenig 50\% igem Alkohol gelöst und auf dem Wasserbade mit $5 \mathrm{ccm}$ einer $5 \%$ igen alkoholischen Pikrinsäurelösung versetzt. Nach dem Erkalten hatten sich vereinzelte Flöckchen am Boden des Bechergläschens ausgeschieden, aber kein Krystall von Lysinpikrat; auch nach Zusatz von mehr 99\% igem Alkohol nicht.

Skraup uud Witt hatten aus der ersten Umfällung ihres Phosphorwolframates, dessen Gewicht ursprünglich $97 \mathrm{~g}$ und nach der Umfällung. $47 \mathrm{~g}$ betrug, 4,2 Sirup erhalten. Durch Lösen dieses Rückstandes in demselben Volumen $50 \%$ igen Alkohols und Vermischen mit dem 11 fachen Volumen einer 5\%igen alkoholischen Pikrinsäure hatten Skraup und Witt ein Gemisch von Krystallen und einem Öl erhalten. Diese Krystalle vereinigt mit den aus weiteren alkoholischen Umfällungen erhaltenen - «die Krystallisation war in dem ersten Filtrat absolut und relativ am allerreichlichsten * - lieferten mehrfach mit Wasser umkrystallisiert bei Skraup und Witt $1 \mathrm{~g}$ reines Lysinpikrat.

Nachdem der von Skraup und Witt befolgte Weg im Kyrinsulfat die Abwesenheit von Lysin ergeben hatte, habe ich das Pikrat weiter nach der von mir vor kurzem ${ }^{1}$ ) gegebenen Vorschrift zur Unterscheidung von Kyrin und dem Gemenge von Arginin und Lysin weiter verarbeitet. Die alkoholische Pikratlösung wurde abgegossen. Die am Boden des Gläschens haftenden Flocken wurden zunächst noch einmal auf Lysin untersucht, indem sie mit wenig Wasser auf dem Wasserbade gelöst wurden

1) Diese Zeitschrift, Bd. XLVIII, S. 65 und 66. 
und die Lösung über Schwefelsäure eingedunstet wurde. Unter dem Mikroskope war wieder kein einziger Krystall sichtbar.

Die abgegossene Pikratlösung wurde unter Zusatz von Pikrinsäure auf dem Wasserbade eingedampft, die Lösung des Rückstandes in $99 \%$ igem Alkohol in Äther verrührt, das in Flocken ausgeschiedene Pikrat abgesaugt, mit Äther gewaschen und über Schwefelsäure getrocknet. Aus der Lösung von $0,25 \mathrm{~g}$ dieses Pikrates in $75 \mathrm{ccm}$ Alkohol $(99 \%$ ) waren weder beim Erkalten noch nach Ätherzusatz Lysinpikrat oder sonstige Krystalle $\mathrm{zu}$ erhalten. Auch nach Zusatz von $0,5 \mathrm{~g}$ Platinchloridchlorwasserstoffsäure in Alkohol gelöst und nach 5 tägigem Stehen im Eisschranke bildete sich kein einziger Krystall des charakteristischen Lysinplatinsalzes. Es war also sicher kein Lysin vorhanden.

Aus dem Filtrate der zweiten Umfällung des Phosphorwolframates aus 80\% igem Alkohol durch Wasser wurde in gleicher Weise wie aus dem Filtrate der ersten Umfällung 0,427 g Rückstand, also ungefähr ebensoviel wie aus dem Filtrate der ersten Umfällung erhalten. Auch in diesem war kein Lysin nachweisbar.

Das zweimal aus Alkohol gefällte Phosphorwolframat wurde, wie früher, in $\mathrm{W}$ asser unter Zusatz von Ammoniak gelöst, diese Lösung mit Barythydrat unter Vermeidung eines wesentlichen Überschusses ausgefällt, das Filtrat nach Zusatz von Ammoniumcarbonat und Filtrieren eingedampft. Von der Lösung des Rückstandes wurde der größere Teil nach schwachem Ansäuern mit Jodwasserstoffsäure, wobei die Lösung völlig klar blieb, tropfenweise mit Kaliumquecksilberjodid versetzt. Es entstand kein Niederschlag, nur eine geringe Opalescenz; ebenso entstand in einer Probe auf Zusatz von mehr Kaliumquecksilberjodid keine Fällung. Die Opalescenz war anderntags verschwunden, dafür war das Gefäß mit einem leichten Anflug bezogen. Nach Abgießen der völlig klaren Lösung und Ausspülen mit etwas Wasser, das ebenfalls klar blieb, wurde das Gefäß mit Alkohol ausgespült und dieser in gewogenem Gläschen eingedampft. Der. Rückstand wog 0,0076 g.

Hiermit in Einklang steht der Befund, daß die von mir 
geprüften Kyrinsulfate ausnahmslos keine Fällung mit Kaliumquecksilberjodid gaben, während die Rohsulfate eine Fällung geben.

Der Rest der Lösung des aus dem zweimal aus der alkoholischen Lösung durch Wasser abgeschiedenen Phosphorwolframate erhaltenen Kyrins wurde eingeengt, mit Schwefelsäure vermischt, so daß die Säurekonzentration dieser Lösung ca. 5\% betrug und in Alkohol verrührt; das feinkörnige Sulfat wurde abgesaugt, mit Alkohol und Äther gewaschen und über Schwefelsäure getrocknet. Es gab die Biuretreaktion deutlich und besaß die Zusammensetzung des Kyrinsulfates. Zur Analyse wurde es im trocknen Luftstrome im Apparat unter Anwendung siedenden Wassers getrocknet.

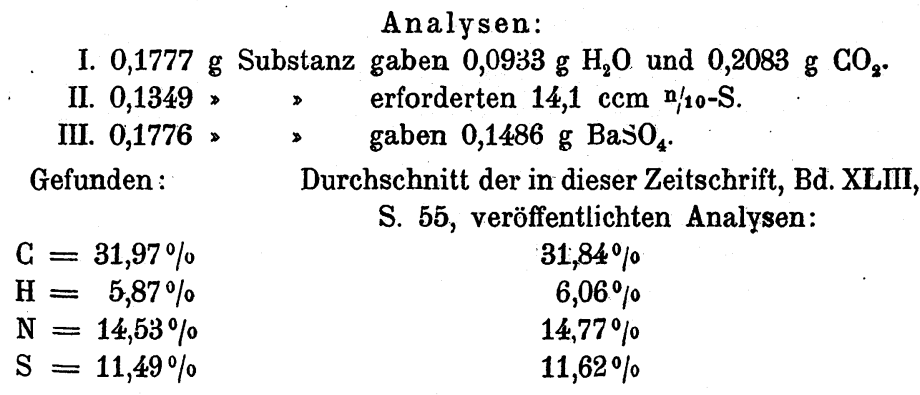

Die Untersuchung des Kyrinphosphorwolframates auf dem von Skraup und Witt eingeschlagenem Wege hat von neuem die Einheitlichkeit des Caseinokyrins bestätigt.

\section{Anwendung der Carbaminoreaktion auf Kyrine.}

Leitet man in die Lösung von Glykokoll bei Gegenwart von Kalkhydrat Kohlensäure, so erhält man das Calciumsalz der Carbaminoessigsäure, der Glykokollcarbonsäure ${ }^{1}$ ). Führt man diese Reaktion unter Einhaltung gewisser, unten näher angégebenen Bedingungen aus, saugt vom überschüssigen Kalkhydrat ab und erhitzt das Filtrat, so zerfällt das glykokollcarbonsaure Calcium in Glykokoll und Calciumcarbonat. Durch Bestimmung des letzteren einerseits und des Stickstoffs im Filtrate anderS. 401.

1) M. Siegfried, Diese Zeitschrift, Bd. XLIV, S. 85, und Bd. XLVI,

Hoppe-Seyler's Zeitschrift f. physiol. Chemie. L. 
seits erhält man Werte, die angeben, wie weit die Reaktion vor sich gegangen ist, d. i. wie viel Glykokoll in das Salz seiner Carbonsäure übergeführt wurde. Dividiert man den Wert für Calciumcarbonat durch das Molekulargewicht desselben und den Wert für Stickstoff durch dessen Atomgewicht, so erhält man, wenn Glykokoll quantitativ in glykokollcarbonsaures Calcium übergeführt wird, das Atomverhältnis :

$$
\mathrm{CO}_{2}: \mathrm{N}=1: 1
$$

Ebenso läßt sich, wenn bekannt ist, wieviel ein Körper mit mehreren N-Atomen Kohlensäure bindet - vorausgesetzt, daß die N-haltigen Gruppen, welche reagieren, quantitativ reagieren - feststellen, wieviel N-Atome der Verbindung die Carbaminoreaktion geben. Es ist hierbei notwendig, daß auch nach dem Aufkochen noch überschüssiges Kalkhydrat in Lösung zugegen ist. Findet man so für das Lysin das Verhältnis $\mathrm{CO}_{2}: \mathrm{N}=1: 1$, so bedeutet dies, daß beide $\mathrm{NH}_{2}$-Gruppen des Lysins die Carbaminoreaktion quantitativ geben. Mit diesen Untersuchungen ist Herr C. Neumann im hiesigen Laboratorium beschäftigt, welcher nicht nur Eiweißspaltungsprodukte, sondern auch Amine und andere stickstoffhaltige Körper auf ihr Verhalten gegenüber der Carbaminoreaktion untersucht, weshalb ich mir dieses Arbeitsgebiet vorbehalten möchte.

Es war zu erwarten, daß Verbindungen, welche bei der Zersetzung Eiweißspaltungsprodukte liefern, wie Eiweißkörper selbst, Peptone, Kyrine, ein kleineres Verhältnis für $\frac{\mathrm{CO}_{2}}{\mathrm{~N}}$ bei der Carbaminoreaktion ergeben, als die Summe ihrer Spaltungsprodukte, da bei der Spaltung sicher N-haltige Gruppen frei werden, welche die Carbaminoreaktion geben, während sie in der Verbindung nicht reagieren. Ich hoffe, daß in dieser Beziehung die Carbaminoreaktion ein Hilfsmittel für Schlüsse auf die Konstitution dieser Verbindungen werden wird, namentlich für Peptone und Kyrine; das wird aber erst möglich sein, wenn das Verhalten möglichst aller hier in Betracht kommenden Aminokörper von bekannter Konstitution untersucht sein wird. Aber schon heute gestattet die Bestimmung des Verhältnisses $\mathrm{CO}_{2}: \mathrm{N}$, z. B. in einem Kyrin und zugleich in der Summe seiner Spal- 
tungsprodukte die Unterschiede zwischen diesem und dem Kyrin von neuem darzutun. Ferner wird diese Methode vielfach geeignet sein, um in einfacher und sicherer Weise zu prüfen, ob ein Körper durch Verdauungsenzyme gespalten wird oder nicht.

Die Bestimmung des Verhältnisses $\mathrm{CO}_{2}: \mathrm{N}$.

2 bis $3 \mathrm{~g}$ Kyrinsulfat werden in Wasser gelöst, bei gewöhnlicher Temperatur mit etwas überschüssigem Barytwasser vermischt, das Filtrat vom Baryumsulfat mit Ammoncarbonat versetzt, das Filtrat vom Baryumcarbonat auf dem Wasserbade vollständig eingedampft; der Rückstand noch einige Male auf dem Wasserbade unter Zusatz von etwas Wasser eingedampft und hierauf zu ungefähr $100 \mathrm{ccm}$ Volumen mit Wasser gelöst. Von dieser Lösung werden 3 bis 4 Bestimmungen ausgeführt. $\mathrm{Zu}$ je $1 / 4$ bis $1 / 3$ der Lösung gibt man einige Tropfen Phenolphtaleinlösung, kühlt in Eiswasser ab, fügt einige Kubikzentimeter Kalkmilch dazu und leitet unter Umschwenken Kohlensäure bis zum Verschwinden der Farbe ein - nicht länger. Darauf gibt man wieder einige Kubikzentimeter Kalkmilch hinzu und leitet wieder unter Umschwenken Kohlensäure bis zum Verschwinden der Farbe ein, gibt noch einmal Kalkmilch hinzu und leitet wieder Kohlensäure bis zum Verschwinden der Farbe ein. Hierauf gibt man wieder etwas Kalkmilch hinzu, schüttelt um, nimmt erst jetzt das Gefäß aus dem Eiswasser und saugt ohne nachzuwaschen ab. - Hat man nach dem letzten Zusatze von Kalkmilch anhaltend umgeschüttelt, ehe man absaugt, so entsteht leicht bei dem nachherigen Aufkochen ein Fehler dadurch, daß sich neben Calciumcarbonat etwas Kalkhydrat abscheidet, da dieses in heißem Wasser schwerer löslich ist als in kaltem. Deshalb verfährt man weiter folgendermaßen: - Das Filtrat wird mit ca. dem doppelten Volumen ausgekochten Wassers versetzt und in einem mit nach abwärts gebogenen Natronkalkrohr versehenen Kölbchen zum Sieden erhitzt. Auch nach dem Kochen muß die Reaktion stark alkalisch sein. Nach Abkühlen wird auf gewogenem Gooch-oder Neubauer-Tiegel abgesaugt, mit kaltem Wasser nachgewaschen. Nach Trocknen bei $120^{\circ}$ gewinnt man den Wert für Calciumcarbonat. 
Das Filtrat wird sogleich mit einem Teil der zur Kjeldahlbestimmung nötigen $20 \mathrm{ccm}$ konzentrierten Schwefelsäure versetzt, im Kjeldahlkolben eingedampft und nach Zusatz des Restes der $20 \mathrm{ccm}$ Schwefelsäure unter Verwendung von Kaliumsulfat und zuletzt Kaliumpermanganat kjeldahlisiert.

Für das Caseinokyrin hat Herr O. Pilz folgende Werte erhalten:

\begin{tabular}{|c|c|c|c|c|c|c|}
\hline & unden: 0,0890 & $\mathrm{aCO}_{3}$ & Gebraucht & $: 20,29$ & $\mathrm{ccm}$ & $n / 10-S$ \\
\hline II. & $: 0,0630$ & - & . & $: 14,49$ & $\nu$ & , \\
\hline III. & $: 0,0910$ & . & ه & :20,66 & , & " \\
\hline IV. & $: 0,0775$ & , & , & 17,67 & $»$ & - \\
\hline
\end{tabular}

Hieraus berechnet sich:

$$
\mathrm{CO}_{2}: \mathrm{N}=1: 2,28 ; 1: 2,30 ; 1: 2,27 ; 1: 2,28 \text {. }
$$

$\mathrm{Da}$ bei diesen schon vor längerer Zeit ausgeführten Bestimmungen das Filtrat vor dem Kochen nicht mit Wasser verdünnt worden war, hat Herr E. Hitschmann unter Anwendung dieser Vorsichtsmaßregel folgende Bestimmungen ausgeführt, deren Resultate mit denen des Herrn Pilz übereinstimmen:

$\begin{array}{rccc} & \mathrm{CaCO}_{8} & \mathrm{ccm} \mathrm{n} / 10-\mathrm{S} & \mathrm{CO}_{2}: \mathrm{N} \\ \text { I. } & 0,1761 & 38,78 & 1: 2,205 \\ \text { II. } & 0,1499 & 33,05 & 1: 2,206 \\ \text { III. } & 0,1626 & 35,65 & 1: 2,194\end{array}$

Herr Pilz hat dann $2 \mathrm{~g}$ Caseinokyrinsulfat durch 12 stündiges Kochen mit der Mischung von $50 \mathrm{ccm}$ Wasser und $25 \mathrm{~g}$ konzentrierter Schwefelsäure zersetzt und für das nach Entfernung der Schwefelsäure mit Barythydrat, Ausfällen des Überschusses dieses mit Ammoncarbonat und wiederholtem Eindampfen auf dem Wasserbade gewonnene Gemenge der Zersetzungsprodukte folgende Werte erhalten:

$\begin{array}{rccc} & \mathrm{CaCO}_{3} & \mathrm{ccm} \mathrm{n} / 10-\mathrm{S} & \mathrm{CO}_{8}: \mathrm{N} \\ \text { I. } & 0,1795 & 26,92 & 1: 1,50 \\ \text { H. } & 0,1245 & 18,92 & 1: 1,52 \\ \text { HI. } & 0,2283 & 33,73 & 1: 1,46 \\ \text { IV. } & 0,1970 & 29,74 & 1: 1,51\end{array}$

Man sieht also, daß das Verhältnis $\mathrm{CO}_{2}: \mathrm{N}$ beim Caseinokyrin ein viel kleineres ist, als das bei seinen Spaltungsprodukten. Im Durchschnitt wurde für den Nenner bei den Zersetzungs- 
produkten gefunden: 1,50, beim Kyrin: 2,25, also für letzteres gerade um $50 \%$ mehr, als für das der Zersetzungsprodukte.

Nimmt man z. B. für das Caseinokyrin 9 Atome $\mathrm{N}$ im Molekül an, so würden - vorausgesetzt, daß die Reaktion quantitativ verläuft - von diesen $9 \mathrm{~N}$-Gruppen 4 die Carbaminoreaktion geben, von den $9 \mathrm{~N}$-Gruppen der Spaltungsprodukte aber $6 \mathrm{~N}$-Gruppen.

Für Fibrinokyrin ${ }^{1}$ ) erhielt Herr Hitschmann folgende Werte:
$\mathrm{CaCO}_{3}$
I. 0,2006
$\mathrm{ccm} n / 10-\mathrm{S}$
$\mathrm{Ca}: \mathrm{N}$
II. 0,2663
52,9
69,37
$1: 2,63$
$1: 2,61$

Das Gemisch der Spaltungsprodukte des Fibrinokyrins gab folgende Werte:
$\mathrm{CaCO}_{8}$
I. 0,2727
ccm n/10-S
$\mathrm{CO}_{2}: \mathrm{N}$
II. 0,2618
38,95
$1: 1,43$
37,20
$1: 1,42$
III. 0,2448
35,40
$1: 1,45$

Beim Fibrinokyrin ist also der Unterschied des Verhältnisses $\mathrm{CO}_{2}: \mathrm{N}$ im Kyrin und der Summe der Spaltungsprodukte noch größer als beim Caseinokyrin.

1) M. Siegfried, Diese Zeitschrift, Bd. XLVIII, S. 67. 